\title{
Time Invariance Violating Nuclear Electric Octupole Moments
}

\author{
V. V. Flambaum ${ }^{1,2}$, D. W. Murray ${ }^{1}$, and S. R. Orton ${ }^{1}$ \\ ${ }^{1}$ School of Physics, University of New South Wales, Sydney, 2052, Australia \\ ${ }^{2}$ ITAMP, Harvard University and the Smithsonian Astrophysical Observatory, 60 Garden Street, \\ Cambridge, Massachusetts 02138
}

\begin{abstract}
The existence of a nuclear electric octupole moment (EOM) requires both parity and time invariance violation. The EOMs of odd $Z$ nuclei that are induced by a particular T- and $\mathrm{P}$-odd interaction are calculated. We compare such octupole moments with the collective EOMs that can occur in nuclei having a static octupole deformation. A nuclear EOM can induce a parity and time invariance violating atomic electric dipole moment, and the magnitude of this effect is calculated. The contribution of a nuclear EOM to such a dipole moment is found, in most cases, to be smaller than that of other mechanisms of atomic electric dipole moment production.
\end{abstract}

PACS numbers: 21.10.Ky, 11.30.Er, 32.80.Ys, 32.10.Dk

Typeset using REVTEX 


\section{INTRODUCTION}

At present, time invariance violation has only been observed indirectly, in the CPviolating decay of neutral K-mesons [四]. Parity and time invariance violating nuclear and atomic multipole moments, such as the magnetic monopole, electric dipole, magnetic quadrupole, and electric octupole moments, are interesting because, if discovered, they would provide further proof of time invariance violation. Even the limits on these moments provide important tests of different models of CP-violation. Parity and time invariance violating nuclear moments induced by T- and P-odd nuclear forces have been discussed, e.g., in Refs. [2]1.

In this paper we consider the electric octupole moments of nuclei. We calculate the EOMs of odd $Z$ nuclei that are induced by a T- and P-odd interaction between the unpaired proton and the nuclear core. Note that nuclei with unpaired neutrons can have an EOM of comparable magnitude due to the polarization of the nuclear core by the T-, P-odd field of

the external neutron. A similar mechanism for the electric dipole and Schiff moments was considered in Ref. [6].

We give values of the collective electric octupole moments of nuclei having a static octupole deformation, using the calculations done in Refs. [8,9]. We also present a calculation of the atomic electric dipole moment (EDM) that would be induced by a nuclear EOM. Finally, we discuss a possible enhancement mechanism for magnetic quadrupole moments (MQMs) in nuclei with octupole deformation.

In the appendix we present simple estimates of the relative sizes of the contributions of various nuclear moments to the atomic EDM. The magnitude of the nuclear EOM is comparable to that of the Schiff moment. However, the contribution of the EOM to the atomic EDM is smaller than the contribution of the Schiff moment (and the magnetic quadrupole moment) since the EOM interacts with higher angular momentum electron states, whose wave functions are suppressed near the nucleus. 


\section{THE HAMILTONIAN OF THE T-, P-ODD NUCLEON-NUCLEUS INTERACTION AND THE RESULTING NUCLEON WAVE FUNCTION}

For a heavy nucleus, the T- and P-odd interaction between a nonrelativistic unpaired nucleon and the nuclear core can be described by the following effective Hamiltonian (see, e.g., Refs. [10,5,7]):

$$
H_{T P}=\eta \frac{G}{2 \sqrt{2} m} \boldsymbol{\sigma} \cdot \nabla \rho
$$

where $\boldsymbol{\sigma}$ is twice the spin operator for this nucleon, $\rho$ is the density of the nuclear core, $G=$ $1.0 \times 10^{-5} / m_{p}{ }^{2}$ is the Fermi constant, $m$ is the mass of the nucleon and $\eta$ is a dimensionless constant that describes the strength of the interaction. In this paper we deal with odd $Z$ nuclei, and so the nucleon involved is the unpaired proton.

Let $U$ be the strong nuclear potential of the core that the unpaired proton moves in. The range of the strong nucleon-nucleon interaction is small. This means that the potential $U(\mathbf{r})$ and the nuclear density $\rho(\mathbf{r})$ will be similar in shape. In fact, we assume that they are approximately proportional: $U(\mathbf{r}) / U(\mathbf{0}) \approx \rho(\mathbf{r}) / \rho(\mathbf{0})$. This allows us to rewrite Eq. (11) in a form that makes it easy to find the perturbed wave function due to $H_{T P}$ (we use the method of Ref. [5]). We obtain

$$
H_{T P} \approx \xi \boldsymbol{\sigma} \cdot \nabla U
$$

where

$$
\xi=\eta \frac{G}{2 \sqrt{2} m_{p}} \frac{\rho(\mathbf{0})}{U(\mathbf{0})}=-2 \times 10^{-21} \eta \mathrm{cm} .
$$

The total potential that the unpaired proton experiences is

$$
\widetilde{U}=U+H_{T P} \approx U(\mathbf{r})+\xi \boldsymbol{\sigma} \cdot \boldsymbol{\nabla} U \approx U(\mathbf{r}+\xi \boldsymbol{\sigma}) .
$$

As a result, if $\psi(\mathbf{r})$ is the proton's wave function when only $U(\mathbf{r})$ is present, the perturbed wave function will be

$$
\widetilde{\psi}(\mathbf{r}) \approx \psi(\mathbf{r}+\xi \boldsymbol{\sigma}) \approx \psi(\mathbf{r})+\xi \boldsymbol{\sigma} \cdot \boldsymbol{\nabla} \psi(\mathbf{r})
$$




\section{THE ELECTRIC OCTUPOLE MOMENT OF A NUCLEUS WITH AN UNPAIRED PROTON}

In this section we calculate the electric octupole moment (EOM) of a nucleus with an unpaired proton using the perturbed wave function obtained above. The electric octupole moment can be written as [see Eq. (A5)]

$$
O_{i j k}=\left\langle\widetilde{\psi}\left|\hat{O}_{i j k}\right| \widetilde{\psi}\right\rangle=e \int \widetilde{\psi}^{\dagger}(\mathbf{r})\left[r_{i} r_{j} r_{k}-\frac{1}{5} r^{2}\left(r_{i} \delta_{j k}+r_{j} \delta_{i k}+r_{k} \delta_{i j}\right)\right] \widetilde{\psi}(\mathbf{r}) d^{3} r .
$$

Note that we use $e>0$. Substituting $\widetilde{\psi}(\mathbf{r})=\psi(\mathbf{r})+\xi \sigma_{m} \frac{\partial \psi}{\partial r_{m}}$ (from Eq. (司)) into the above integral and expanding gives

$$
O_{i j k}=2 e \xi \int \frac{\partial \psi^{\dagger}}{\partial r_{m}} \sigma_{m}\left[r_{i} r_{j} r_{k}-\frac{1}{5} r^{2}\left(r_{i} \delta_{j k}+r_{j} \delta_{i k}+r_{k} \delta_{i j}\right)\right] \psi d^{3} r
$$

Notice that we have discarded the $\xi^{2}$ term and that the term not containing $\xi$ vanishes, as it is an integral of an odd function of $\mathbf{r}$. Applying integration by parts to the above integral gives

$$
\begin{gathered}
O_{i j k}=-\frac{1}{5} e \xi\langle\psi| 5\left(\hat{r}_{i} \hat{r}_{j} \hat{\sigma}_{k}+\hat{r}_{i} \hat{r}_{k} \hat{\sigma}_{j}+\hat{r}_{j} \hat{r}_{k} \hat{\sigma}_{i}\right)-\hat{r}^{2}\left(\hat{\sigma}_{i} \delta_{j k}+\hat{\sigma}_{j} \delta_{i k}+\hat{\sigma}_{k} \delta_{i j}\right) \\
-2 \hat{r}_{m} \hat{\sigma}_{m}\left(\hat{r}_{i} \delta_{j k}+\hat{r}_{j} \delta_{i k}+\hat{r}_{k} \delta_{i j}\right)|\psi\rangle .
\end{gathered}
$$

We can also write the octupole moment tensor in another form. Since it is a symmetric, irreducible (traceless) third rank tensor and the nuclear angular momentum $\mathbf{I}$ is the only quantity which defines a direction in the system, the EOM tensor must be in the form of the most general symmetric, irreducible third rank tensor that can be formed from the components of $\hat{\mathbf{I}}$. That is

$$
O_{i j k}=\left\langle\widetilde{\psi}\left|\hat{O}_{i j k}\right| \widetilde{\psi}\right\rangle
$$

where

$$
\begin{gathered}
\hat{O}_{i j k}=A\left[\hat{I}_{i} \hat{I}_{j} \hat{I}_{k}+\hat{I}_{j} \hat{I}_{k} \hat{I}_{i}+\hat{I}_{k} \hat{I}_{i} \hat{I}_{j}+\hat{I}_{k} \hat{I}_{j} \hat{I}_{i}+\hat{I}_{j} \hat{I}_{i} \hat{I}_{k}+\hat{I}_{i} \hat{I}_{k} \hat{I}_{j}\right. \\
\left.-\frac{6 I(I+1)-2}{5}\left(\hat{I}_{i} \delta_{j k}+\hat{I}_{j} \delta_{i k}+\hat{I}_{k} \delta_{i j}\right)\right]
\end{gathered}
$$


and $A$ is some constant. The factor of $-[6 I(I+1)-2] / 5$ follows from the requirement of tracelessness $\left(O_{i i j}=O_{i j i}=O_{j i i}=0\right)$. The quantity which is usually referred to as the octupole moment is $\mathcal{O}$, which is the $O_{z z z}$ component for the nuclear state having angular momentum projection $I_{z}=I$. This is the quantity which we will calculate. We can write $A$ in terms of $\mathcal{O}$ using Eqs. (9) and (10), with $i=j=k=z$. This gives

$$
\begin{gathered}
\hat{O}_{i j k}=\frac{5 \mathcal{O}}{6 I(I-1)(2 I-1)}\left[\hat{I}_{i} \hat{I}_{j} \hat{I}_{k}+\hat{I}_{j} \hat{I}_{k} \hat{I}_{i}+\hat{I}_{k} \hat{I}_{i} \hat{I}_{j}+\hat{I}_{k} \hat{I}_{j} \hat{I}_{i}+\hat{I}_{j} \hat{I}_{i} \hat{I}_{k}+\hat{I}_{i} \hat{I}_{k} \hat{I}_{j}\right. \\
\left.-\frac{6 I(I+1)-2}{5}\left(\hat{I}_{i} \delta_{j k}+\hat{I}_{j} \delta_{i k}+\hat{I}_{k} \delta_{i j}\right)\right] .
\end{gathered}
$$

We now have two expressions for $O_{i j k}$ : Eq. (8) and Eq. (11) (via Eq. (9)). We calculate $\mathcal{O}$ by operating on both of these equations with $\hat{I}_{i} \hat{I}_{j}$ on the left and $\hat{I}_{k}$ on the right and equating the results.

To evaluate the results of these operations, the following commutation relations are required: $\left[\hat{r}_{i}, \hat{\sigma}_{j}\right]=0,\left[\hat{I}_{i}, \hat{r}_{j}\right]=i \varepsilon_{i j k} \hat{r}_{k}$, and $\left[\hat{I}_{i}, \hat{\sigma}_{j}\right]=i \varepsilon_{i j k} \hat{\sigma}_{k}$. (Note that $\left.\hat{I}_{i}=\hat{l}_{i}+\hat{\sigma}_{i} / 2.\right)$ Other useful relations are $\left[\hat{I}_{i}, \hat{I}_{j} \hat{r}_{j}\right]=\left[\hat{I}_{i}, \hat{I}_{j} \hat{\sigma}_{j}\right]=\left[\hat{I}_{i}, \hat{\sigma}_{j} \hat{r}_{j}\right]=0$ and $\varepsilon_{i j k} A_{i} A_{j}=\frac{1}{2} \varepsilon_{i j k} A_{i} A_{j}-$ $\frac{1}{2} \varepsilon_{i j k} A_{j} A_{i}=\frac{1}{2} \varepsilon_{i j k}\left[A_{i}, A_{j}\right]$, for an operator $A_{i}$. The commutation relations are used to rearrange the operators so that pairs with the same indices are adjacent.

Applying the operators to Eq. (8) gives

$$
\begin{aligned}
\left\langle\widetilde{\psi}\left|\hat{I}_{i} \hat{I}_{j} \hat{O}_{i j k} \hat{I}_{k}\right| \widetilde{\psi}\right\rangle=-\frac{1}{5} e \xi\langle\psi| & 5 \hat{I}_{i} \hat{I}_{j}\left(\hat{r}_{i} \hat{r}_{j} \hat{\sigma}_{k}+\hat{r}_{i} \hat{r}_{k} \hat{\sigma}_{j}+\hat{r}_{j} \hat{r}_{k} \hat{\sigma}_{i}\right) \hat{I}_{k}-\hat{I}_{i} \hat{I}_{j} \hat{r}^{2}\left(\hat{\sigma}_{i} \delta_{j k}+\hat{\sigma}_{j} \delta_{i k}+\hat{\sigma}_{k} \delta_{i j}\right) \hat{I}_{k} \\
& -2 \hat{I}_{i} \hat{I}_{j} \hat{r}_{m} \hat{\sigma}_{m}\left(\hat{r}_{i} \delta_{j k}+\hat{r}_{j} \delta_{i k}+\hat{r}_{k} \delta_{i j}\right) \hat{I}_{k}|\psi\rangle \\
=-\frac{1}{5} e \xi\left\langle r^{2}\right\rangle\{ & \left\langle I, I_{z}, l\left|(\hat{\boldsymbol{\sigma}} \cdot \hat{\mathbf{n}})\left[\frac{5}{4}(\hat{\mathbf{I}} \cdot \hat{\boldsymbol{\sigma}})-\hat{I}^{2}\right](\hat{\boldsymbol{\sigma}} \cdot \hat{\mathbf{n}})\right| I, I_{z}, l\right\rangle \\
& \left.+\left\langle I, I_{z}, l\left|\frac{7}{2}(\hat{\mathbf{I}} \cdot \hat{\boldsymbol{\sigma}})-3 \hat{I}^{2}(\hat{\mathbf{I}} \cdot \hat{\boldsymbol{\sigma}})+1-2 \hat{I}^{2}\right| I, I_{z}, l\right\rangle\right\}
\end{aligned}
$$

where $\hat{\mathbf{n}}=\hat{\mathbf{r}} / r$. Here the facts that $\hat{\mathbf{I}} \cdot \hat{\mathbf{n}}=(\hat{\mathbf{l}}+\hat{\boldsymbol{\sigma}} / 2) \cdot \hat{\mathbf{n}}=(\hat{\boldsymbol{\sigma}} \cdot \hat{\mathbf{n}}) / 2$ and $(\hat{\boldsymbol{\sigma}} \cdot \hat{\mathbf{n}})^{2}=1$ [11 were used. The radial and angular parts of the operators have been separated in Eq. (12); $\left\langle r^{2}\right\rangle$ is the expectation value of $r^{2}$ and $\left|I, I_{z}, l\right\rangle$ is the angular part of $|\psi\rangle$. The second matrix element in Eq. (12) is

$$
\left\langle I, I_{z}, l\left|\frac{7}{2}(\hat{\mathbf{I}} \cdot \hat{\boldsymbol{\sigma}})-3 \hat{I}^{2}(\hat{\mathbf{I}} \cdot \hat{\boldsymbol{\sigma}})+1-2 \hat{I}^{2}\right| I, I_{z}, l\right\rangle=\left[\frac{7}{2}-3 I(I+1)\right]\left(\frac{1}{2}-\kappa\right)+1-2 I(I+1),
$$


where we have used the fact that

$$
\begin{aligned}
(\hat{\mathbf{I}} \cdot \hat{\boldsymbol{\sigma}})\left|I, I_{z}, l\right\rangle & =[I(I+1)-l(l+1)+3 / 4]\left|I, I_{z}, l\right\rangle \\
& =(1 / 2-\kappa)\left|I, I_{z}, l\right\rangle,
\end{aligned}
$$

with

$$
\kappa=(I+1 / 2)(-1)^{I+1 / 2-l} .
$$

To evaluate the first matrix element in Eq. (12), we use the following identity:

$$
(\hat{\boldsymbol{\sigma}} \cdot \hat{\mathbf{n}})\left|I, I_{z}, l\right\rangle=-\left|I, I_{z}, \widetilde{l}\right\rangle
$$

where $\widetilde{l}=2 I-l$. We then have

$$
\begin{aligned}
\left\langle I, I_{z}, l\left|(\hat{\boldsymbol{\sigma}} \cdot \hat{\mathbf{n}})\left[\frac{5}{4}(\hat{\mathbf{I}} \cdot \hat{\boldsymbol{\sigma}})-\hat{I}^{2}\right](\hat{\boldsymbol{\sigma}} \cdot \hat{\mathbf{n}})\right| I, I_{z}, l\right\rangle & =\left\langle I, I_{z}, \widetilde{l}\left|\frac{5}{4}(\hat{\mathbf{I}} \cdot \hat{\boldsymbol{\sigma}})-\hat{I}^{2}\right| I, I_{z}, \widetilde{l}\right\rangle \\
& =\frac{5}{4}\left(\frac{1}{2}+\kappa\right)-I(I+1) .
\end{aligned}
$$

(Note that $(\hat{\mathbf{I}} \cdot \hat{\boldsymbol{\sigma}})\left|I, I_{z}, \widetilde{l}\right\rangle=(1 / 2+\kappa)\left|I, I_{z}, \widetilde{l}\right\rangle$.) Using these results in Eq. (12) gives

$$
\left\langle\widetilde{\psi}\left|\hat{I}_{i} \hat{I}_{j} \hat{O}_{i j k} \hat{I}_{k}\right| \widetilde{\psi}\right\rangle=-\frac{3}{5} e \xi\left\langle r^{2}\right\rangle\left(\kappa-\frac{3}{2}\right)\left(I-\frac{1}{2}\right)\left(I+\frac{3}{2}\right) .
$$

Now we find another form of the left hand side of the above equation by operating on Eq. (111). We have

$$
\begin{gathered}
\left\langle\widetilde{\psi}\left|\hat{I}_{i} \hat{I}_{j} \hat{O}_{i j k} \hat{I}_{k}\right| \widetilde{\psi}\right\rangle=\frac{5 \mathcal{O}}{6 I(I-1)(2 I-1)}\langle\widetilde{\psi}| \hat{I}_{i} \hat{I}_{j}\left(\hat{I}_{i} \hat{I}_{j} \hat{I}_{k}+\hat{I}_{j} \hat{I}_{k} \hat{I}_{i}+\hat{I}_{k} \hat{I}_{i} \hat{I}_{j}+\hat{I}_{k} \hat{I}_{j} \hat{I}_{i}+\hat{I}_{j} \hat{I}_{i} \hat{I}_{k}+\hat{I}_{i} \hat{I}_{k} \hat{I}_{j}\right) \hat{I}_{k} \\
-\frac{6 I(I+1)-2}{5} \hat{I}_{i} \hat{I}_{j}\left(\hat{I}_{i} \delta_{j k}+\hat{I}_{j} \delta_{i k}+\hat{I}_{k} \delta_{i j}\right) \hat{I}_{k}|\widetilde{\psi}\rangle .
\end{gathered}
$$

This can be evaluated using the equation $\left[\hat{I}_{i}, \hat{I}_{j}\right]=i \varepsilon_{i j k} \hat{I}_{k}$ to bring operators with the same indices together and $\varepsilon_{i j k} \hat{I}_{i} \hat{I}_{j}=\frac{i}{2} \varepsilon_{i j k} \varepsilon_{i j p} \hat{I}_{p}=i \hat{I}_{k}$. The result is

$$
\left\langle\widetilde{\psi}\left|\hat{I}_{i} \hat{I}_{j} \hat{O}_{i j k} \hat{I}_{k}\right| \widetilde{\psi}\right\rangle=\mathcal{O}(I+1)(I+3 / 2)(I+2) .
$$

Equating this result with Eq. (18) gives the following result for the octupole moment:

$$
\mathcal{O}_{\text {sing }}=\frac{-3(\kappa-3 / 2)(I-1 / 2)}{5(I+1)(I+2)}\left\langle r^{2}\right\rangle e \xi,
$$


(The subscript refers to the fact that this octupole moment is due to a single particle, as opposed to a collective octupole moment.) The expectation value for $r^{2}$ can be approximated as [5]

$$
\left\langle r^{2}\right\rangle \approx \frac{3}{5} r_{0}^{2} A^{2 / 3}
$$

to an accuracy of about $10 \%$, where $r_{0}=1.1 \mathrm{fm}$ and $A$ is the mass number of the nucleus. Using the value of $r_{0}$ and Eqs. (3) and (15) gives

$$
\mathcal{O}_{\text {sing }} \approx 8.7 \times 10^{-9} A^{2 / 3} \eta e(\mathrm{fm})^{3} \times \begin{cases}\frac{-(I-1 / 2)}{I+1} & \text { for } I=l+1 / 2 \\ \frac{(I-1 / 2)(I-1)}{(I+1)(I+2)} & \text { for } I=l-1 / 2\end{cases}
$$

Observe that $\mathcal{O}_{\text {sing }}=0$ for $I=1 / 2$. This is to be expected because $\hat{O}_{i j k}$ is a third rank tensor, and so applying the triangle rule for the addition of angular momenta to Eq. (9) gives the result that nuclei having angular momentum less than $3 / 2$ cannot have an octupole moment. Values of $\mathcal{O}_{\text {sing }}$ for various nuclei are given in table $\llbracket$, in terms of the parameter $\eta$.

\section{COLLECTIVE ELECTRIC OCTUPOLE MOMENTS IN NUCLEI WITH STATIC OCTUPOLE DEFORMATION}

In Refs. [8,9], a mechanism was suggested by which parity and time invariance violating interactions can produce collective $\mathrm{T}$ - and P-odd multipole moments in even-odd nuclei having a static octupole deformation [i.e. electric octupole moments in their intrinsic (or body-fixed) reference frames]. Such a deformation has been shown to exist for nuclei in the $\mathrm{Ra}-\mathrm{Th}$ and $\mathrm{Ba}-\mathrm{Sm}$ regions (for a review see, e.g., [12]). A similar mechanism, for the enhancement of the intrinsic electron EDM and other T-, P-odd interactions in polar molecules, was suggested in Ref. [13]. Below, we explain the mechanism by which a collective nuclear EOM can be produced and provide values of this EOM for various nuclei. 


\section{A. Parity and time invariance and octupole moments in the laboratory frame}

An electric octupole moment can exist in the nucleus's intrinsic frame without parity or time invariance violation. Yet if parity and time invariance hold, the expectation value of the octupole moment in the laboratory reference frame will be zero.

Consider $|I M K\rangle$ and $|I M-K\rangle$, which are two almost degenerate states of the nucleus in the laboratory frame. $I$ is the angular momentum of the nucleus, $M$ is its projection onto the $z$-axis, and $K$ is its projection onto the axis of symmetry of the deformed nuclear core (the $z^{\prime}$-axis). ( $\mathbf{I}$ is the sum of the unpaired nucleon's angular momentum, $\mathbf{j}$ and the nuclear core's orbital angular momentum, R.) These states can be written in terms of intrinsic states as

$$
|I M \pm K\rangle=\sqrt{\frac{2 I+1}{4 \pi}} D_{M \pm K}^{I}(\phi, \theta, 0) \psi_{ \pm K}\left(\mathbf{r}^{\prime}\right) \chi_{\text {core }}
$$

where $D_{M \pm K}^{I}(\phi, \theta, 0)$ is a Wigner $D$-function (see, e.g., [14.15]), $\chi_{\text {core }}$ is the wave function of the nuclear core in the intrinsic frame, and $\psi_{ \pm K}\left(\mathbf{r}^{\prime}\right)$ is the wave function of the unpaired nucleon in the intrinsic frame, with a $z^{\prime}$ angular momentum projection of $\pm K$. (In the intrinsic frame, the nuclear axis plays the role of the usual " $z$-axis" in Quantum Mechanics.) Note that $\mathbf{j}$ and $\mathbf{I}$ have the same $z^{\prime}$ projection.

$|I M K\rangle$ and $|I M-K\rangle$ do not have good parity, as $K$ changes sign under a parity transformation. However, the following states do, and they form a parity doublet:

$$
\psi^{ \pm}=\frac{1}{\sqrt{2}}(|I M K\rangle \pm|I M-K\rangle) .
$$

For these good parity states $\left\langle\psi^{ \pm}|\mathbf{I} \cdot \mathbf{n}| \psi^{ \pm}\right\rangle=0$ because $K$ and $-K$ have equal probabilities and this means that there is no average orientation of the nuclear axis in the laboratory frame $\left(\left\langle\psi^{ \pm}|\mathbf{n}| \psi^{ \pm}\right\rangle=\mathbf{0}\right)$. This is a consequence of time invariance and parity conservation since the correlation $\mathbf{I} \cdot \mathbf{n}$ is T-, P-odd. As a result of $\left\langle\psi^{ \pm}|\mathbf{n}| \psi^{ \pm}\right\rangle=\mathbf{0}$, the mean value of the octupole moment (whose orientation is determined by the direction of the nuclear axis) is zero in the laboratory frame. 
Now, a T- and P-odd interaction, $H_{T P}$ will mix the members of the parity doublet $\left(\psi^{+}\right.$ and $\left.\psi^{-}\right)$. The admixed wave function of the predominantly positive parity member of the doublet will be $\psi=\psi^{+}+\alpha \psi^{-}$or

$$
\psi=\frac{1}{\sqrt{2}}[(1+\alpha)|I M K\rangle+(1-\alpha)|I M-K\rangle],
$$

where $\alpha$ is a mixing coefficient:

$$
\alpha=\frac{\left\langle\psi^{-}\left|H_{T P}\right| \psi^{+}\right\rangle}{E_{+}-E_{-}} .
$$

$E_{+}-E_{-}$is the energy splitting between the members of the parity doublet. The interaction $H_{T P}$ is given by Eq. (四). (A similar expression can be obtained for the predominantly negative parity member of the doublet.) This mixing yields, on average, an orientation of the nuclear axis along the direction of the angular momentum:

$$
\langle\psi|\mathbf{I} \cdot \mathbf{n}| \psi\rangle=\langle\psi|\hat{K}| \psi\rangle=2 \alpha K
$$

and this means that the octupole moment need no longer vanish in the laboratory frame.

\section{B. The magnitude of the collective octupole moment}

The collective EOM in the laboratory frame was derived in [8,9], with the following result:

$$
\mathcal{O}_{\text {coll }} \approx \frac{4}{5} \frac{I(I-1)(I-1 / 2)}{(I+1)(I+2)(I+3 / 2)} \alpha O_{3, \text { intr }} .
$$

Once again, observe that $\mathcal{O}_{\text {coll }}=0$ for $I=1 / 2 . O_{3, \text { intr }}$ refers to the octupole moment in the intrinsic frame $\left(O_{z z z} \equiv \frac{2}{5} O_{3}\right)$ and is given by [16, 17]:

$$
O_{3, \text { intr }}=e Z R_{0}{ }^{3} \frac{3}{2 \sqrt{7 \pi}}\left(\beta_{3}+\frac{2}{3} \sqrt{\frac{5}{\pi}} \beta_{2} \beta_{3}+\frac{15}{11 \sqrt{\pi}} \beta_{3} \beta_{4}+\ldots\right),
$$

where $R_{0}=r_{0} A^{1 / 3}\left(r_{0}=1.1 \mathrm{fm}\right) . \beta_{2}, \beta_{3}$, and $\beta_{4}$ are parameters that describe the nuclear deformation; the surface of a deformed nucleus is 


$$
R=R_{0}\left[1+\sum_{l=1}^{\infty} \beta_{l} Y_{l 0}(\theta, \phi)\right]
$$

We will first present an order of magnitude estimate of $\alpha . \hat{K}=\mathbf{I} \cdot \mathbf{n}$ and $H_{T P}$ are both T-, P-odd pseudoscalars. Therefore, $\left\langle\psi_{+K}\left|H_{T P}\right| \psi_{+K}\right\rangle \propto K$ and so $\left\langle\psi_{-K}\left|H_{T P}\right| \psi_{-K}\right\rangle=$ $-\left\langle\psi_{+K}\left|H_{T P}\right| \psi_{+K}\right\rangle$ (this fact can be easily supported by model calculations). Using this fact and Eqs. (24) and (25) we get $\left\langle\psi^{-}\left|H_{T P}\right| \psi^{+}\right\rangle=\left\langle\psi_{+K}\left|H_{T P}\right| \psi_{+K}\right\rangle$. If $\psi_{+K}$ were a good parity state this matrix element would be zero. However, due to the perturbation caused by the static octupole deformation of the nucleus $\left(V_{3}\right)$, it is a combination of the opposite parity spherical orbitals $\psi_{1,+K}$ and $\psi_{2,+K}$ (e.g., $p_{3 / 2}$ and $d_{3 / 2}$ ):

$$
\begin{gathered}
\psi_{+K}=\psi_{1,+K}+\gamma \psi_{2,+K}, \\
\gamma=\frac{\left\langle\psi_{2,+K}\left|V_{3}\right| \psi_{1,+K}\right\rangle}{E_{1}-E_{2}}, \\
\psi_{1,+K}=R_{1}\left(r^{\prime}\right) \Omega_{j, l,+K}\left(\theta^{\prime}, \phi^{\prime}\right), \\
\psi_{2,+K}=R_{2}\left(r^{\prime}\right) \Omega_{j, \widetilde{l},+K}\left(\theta^{\prime}, \phi^{\prime}\right)=-R_{2}\left(r^{\prime}\right)\left(\boldsymbol{\sigma} \cdot \mathbf{n}^{\prime}\right) \Omega_{j, l,+K}\left(\theta^{\prime}, \phi^{\prime}\right),
\end{gathered}
$$

where $\tilde{l}=2 j-l$. (Of course there will also be an admixture of other opposite parity states having different values of $j$. We neglect these states for simplicity.) Therefore, we have

$$
\alpha=\frac{\left\langle\psi_{+K}\left|H_{T P}\right| \psi_{+K}\right\rangle}{E_{+}-E_{-}}=2 \gamma \frac{\left\langle\psi_{1,+K}\left|H_{T P}\right| \psi_{2,+K}\right\rangle}{E_{+}-E_{-}}
$$

To estimate $\gamma$ we must first derive the form of $V_{3}$. As in Sec. II, let $U$ be the strong nuclear potential that the unpaired nucleon moves in. $V_{3}$ is the difference between the potentials with the octupole deformation present, $U_{\text {pres }}$ and absent, $U_{\text {abs }}$. Once again we have $U\left(\mathbf{r}^{\prime}\right) \approx U(\mathbf{0}) \rho\left(\mathbf{r}^{\prime}\right) / \rho(\mathbf{0})$. We also make the approximation that $\rho\left(\mathbf{r}^{\prime}\right) / \rho(\mathbf{0}) \approx \theta\left(r^{\prime}-R\right)$, where $R$ is the nuclear radius and $\theta(x)=1$ for $x<0$ and $\theta(x)=0$ for $x>0$. For an octupole deformed nucleus we have $R=R_{0}\left(1+\beta_{3} Y_{30}\right)$ and so

$$
V_{3}=U_{\text {pres }}-U_{\mathrm{abs}} \approx U(\mathbf{0})\left[\theta\left(r^{\prime}-R_{0}-\beta_{3} Y_{30} R_{0}\right)-\theta\left(r^{\prime}-R_{0}\right)\right] \approx U(\mathbf{0}) R_{0} \beta_{3} \delta\left(r^{\prime}-R_{0}\right) Y_{30}
$$


where we have expanded $\theta$ in a Taylor series, using $\frac{d \theta}{d x}=-\delta(x)$. Using Eq. (33) we then have

$$
|\gamma| \approx\left|U(\mathbf{0}) \beta_{3} R_{1}\left(R_{0}\right) R_{2}\left(R_{0}\right) R_{0}^{3} \int \Omega_{2}^{\dagger} Y_{30} \Omega_{1} d \Omega^{\prime}\left(E_{1}-E_{2}\right)^{-1}\right| \sim \beta_{3}
$$

where we have used $R_{1}\left(R_{0}\right) R_{2}\left(R_{0}\right) \approx 1.4 / R_{0}{ }^{3}$ [16], $|U(\mathbf{0})| \approx 50 \mathrm{MeV},\left|E_{1}-E_{2}\right| \approx 5 \mathrm{MeV}$, and $\int \Omega_{2}^{\dagger} Y_{30} \Omega_{1} d \Omega^{\prime} \sim 0.05$.

Finally, we must estimate the matrix element between the spherical orbitals, $\left\langle\psi_{1,+K}\left|H_{T P}\right| \psi_{2,+K}\right\rangle$. Using Eq. (1) for the form of $H_{T P}$ and $\rho\left(r^{\prime}\right)=\theta\left(r^{\prime}-R_{0}\right) /\left(\frac{4}{3} \pi r_{0}^{3}\right)$ we get

$$
H_{T P}=-\eta \frac{3 G}{8 \pi \sqrt{2} m r_{0}^{3}}\left(\boldsymbol{\sigma} \cdot \mathbf{n}^{\prime}\right) \delta\left(r^{\prime}-R_{0}\right) \text {. }
$$

Using Eq. (34) and $\left(\boldsymbol{\sigma} \cdot \mathbf{n}^{\prime}\right)^{2}=1$ gives

$$
\left\langle\psi_{1,+K}\left|H_{T P}\right| \psi_{2,+K}\right\rangle=\eta \frac{3 G}{8 \pi \sqrt{2} m r_{0}^{3}} R_{1}\left(R_{0}\right) R_{2}\left(R_{0}\right) R_{0}^{2} \approx \frac{\eta}{A^{1 / 3}} 1 \mathrm{eV} .
$$

Using $\left|E_{+}-E_{-}\right| \sim 50 \mathrm{keV}$ (see, e.g., 8,9]), $\beta_{3} \approx 0.1$ (see, e.g., [18]), and Eqs. (35), (37), and (39) gives (for $A \approx 225)|\alpha| \sim 2 \beta_{3} A^{-1 / 3} \eta \mathrm{eV} /\left|E_{+}-E_{-}\right| \sim 7 \times 10^{-7} \eta$. This provides the following estimate for the collective EOM:

$$
\left|\mathcal{O}_{\text {coll }}\right| \sim 0.05 e \beta_{3}^{2} Z A^{2 / 3} r_{0}^{3} \eta \mathrm{eV} /\left|E_{+}-E_{-}\right| \sim 4 \times 10^{-5} \eta e(\mathrm{fm})^{3}
$$

We see that the collective EOM is two orders of magnitude larger than the EOM due to unpaired protons.

We can do a more accurate calculation of the EOM using Refs. [8.9, where the mixing coefficients, $\alpha$ for various nuclei were calculated. We use the values from [9] that were calculated using the Woods-Saxon potential. We took the values of the $\beta_{i}$ parameters from 18 and the nuclei's angular momenta were taken from [19,17. The results are shown in table [1] for various nuclei.

Note the large value of ${ }^{229} \mathrm{~Pa}$ 's collective octupole moment. This is due to its large value of $\alpha$, which is caused by the small energy splitting between the members of its parity doublet 
[8.9]. The possible existence of a static octupole deformation in ${ }^{229} \mathrm{~Pa}$ was stated in [20]. However, more recent papers cast doubt on the existence of such a deformation (see, e.g., [21,22]). Therefore, the result given for ${ }^{229} \mathrm{~Pa}$ must be understood as being conditional on it having a static octupole deformation.

\section{THE ATOMIC ELECTRIC DIPOLE MOMENT INDUCED BY A NUCLEAR ELECTRIC OCTUPOLE MOMENT}

In this section we consider the electric dipole moment of an atom that is induced by a nuclear electric octupole moment. The electric potential, $\phi(\mathbf{r})$ outside an arbitrary charge distribution can be expanded in terms of spherical harmonics as (see, e.g., [23])

$$
\phi(\mathbf{r})=\sum_{l=0}^{\infty} \sum_{m=-l}^{l} \frac{4 \pi}{2 l+1} q_{l m} \frac{Y_{l m}(\theta, \phi)}{r^{l+1}} .
$$

Here we neglect that part of the potential that comes from the screening of the nucleus's Coulomb field by the atomic electrons as it is not important in the octupole potential (see the appendix). The $q_{l m}$ are spherical electric multipole moments and they can be written in terms of the charge distribution $\rho_{c}\left(\mathbf{r}^{\prime}\right)$ as follows [23:

$$
q_{l m}=\int Y_{l m}^{*}\left(\theta^{\prime}, \phi^{\prime}\right) r^{\prime l} \rho_{c}\left(\mathbf{r}^{\prime}\right) d^{3} r^{\prime}
$$

The octupole term of the above electric potential is

$$
\phi^{(3)}(\mathbf{r})=\frac{4 \pi}{7} q_{30} \frac{1}{r^{4}} Y_{30}(\theta, \phi),
$$

where we have only taken the $m=0$ term, as we will be calculating the matrix element of the perturbation between states with the same angular momentum projections. We can write this in terms of the octupole moment by using the relation $q_{30}=\int Y_{30}(\theta, \phi) r^{3} \rho_{c}(\mathbf{r}) d^{3} r=$

$\frac{5}{4} \sqrt{\frac{7}{\pi}} O_{z z z}$. The potential energy, $U_{\text {oct }}$ of an electron (of charge $-e$ ) in $\phi^{(3)}(\mathbf{r})$ will then be

$$
U_{\text {oct }}=-5 \sqrt{\frac{\pi}{7}} e \mathcal{O} \frac{1}{r^{4}} Y_{30}(\theta, \phi)
$$

(for the nuclear $I_{z}=I$ state). 
Using perturbation theory, the electric dipole moment of, for example, an atom with one electron above closed subshells induced by $U_{\text {oct }}$ can be written as

$$
d_{z}=-e\left\langle\widetilde{\psi}\left|r_{z}\right| \widetilde{\psi}\right\rangle=-2 e \sum_{\left|k_{2}\right\rangle} \frac{\left\langle k_{1}\left|r_{z}\right| k_{2}\right\rangle\left\langle k_{2}\left|U_{\mathrm{oct}}\right| k_{1}\right\rangle}{E_{k_{1}}-E_{k_{2}}}
$$

where $\widetilde{\psi}$ denotes the perturbed atomic wave function, $\left|k_{1}\right\rangle=\left|n_{1}, j_{1}, l_{1}, m\right\rangle$ is the unperturbed single-electron ground state, and $\left\{\left|k_{2}\right\rangle\right\}$ is the set of states with which $\left|k_{1}\right\rangle$ is mixed by the perturbation.

According to the triangle rule for the addition of angular momenta, $\left\langle k_{1}\left|r_{z}\right| k_{2}\right\rangle$ can only have a nonzero value if $\left|j_{1}-j_{2}\right| \leq 1 \leq j_{1}+j_{2}$. Similarly, for $\left\langle k_{2}\left|U_{\text {oct }}\right| k_{1}\right\rangle$ to be nonzero, we must have $\left|j_{1}-j_{2}\right| \leq 3 \leq j_{1}+j_{2}$. This implies that the following conditions need to be satisfied for the dipole moment to be nonzero:

$$
\left|j_{1}-j_{2}\right| \leq 1 \text { and } j_{1}+j_{2} \geq 3
$$

The lowest pair of values that satisfies this condition is $j_{1}=3 / 2$ and $j_{2}=3 / 2$. Therefore, $s$ states cannot contribute to the dipole moment induced by the nuclear EOM. Also, one of $l_{1}$ and $l_{2}$ must be even and the other odd, since the electric dipole moment is a parity nonconserving effect.

We will carry out a relativistic calculation of the matrix element of $U_{\text {oct }}$ between the single-electron states $\left|n_{1}, j_{1}, l_{1}, m\right\rangle$ and $\left|n_{2}, j_{2}, l_{2}, m\right\rangle$. (Note that although we wrote Eq. (45) for an atom with one electron above closed subshells, this single-electron state matrix element can be used for all atoms to compare various sources of atomic EDMs.) The relativistic wave function of an electron is (see, e.g., [15])

$$
\psi_{n j l m}=\left(\begin{array}{c}
f_{n j l}(r) \Omega_{j l m}(\theta, \phi) \\
g_{n j l}(r) i(-\boldsymbol{\sigma} \cdot \mathbf{n}) \Omega_{j l m}(\theta, \phi)
\end{array}\right),
$$

where $\boldsymbol{\sigma}$ is twice the spin operator of this electron and $\mathbf{n}=\mathbf{r} / r$. Evaluating the matrix element (using $(\boldsymbol{\sigma} \cdot \mathbf{n})^{2}=1$ ) gives

$$
\left\langle n_{1} j_{1} l_{1} m\left|U_{\text {oct }}\right| n_{2} j_{2} l_{2} m\right\rangle=-5 \sqrt{\frac{\pi}{7}} e \mathcal{O}\left\langle j_{1} l_{1} m\left|Y_{30}\right| j_{2} l_{2} m\right\rangle T
$$


where $T$ is a radial integral:

$$
T=\int_{0}^{\infty} \frac{1}{r^{4}}\left(f_{n_{1} j_{1} l_{1}} f_{n_{2} j_{2} l_{2}}+g_{n_{1} j_{1} l_{1}} g_{n_{2} j_{2} l_{2}}\right) r^{2} d r .
$$

Because of the factor of $1 / r^{4}$ in the above integral, most of the contribution to $T$ comes from small values of $r$. This allows us to use the following expressions for $f_{n j l}$ and $g_{n j l}$, for $r \ll a / Z^{1 / 3}[7]:$

$$
\begin{aligned}
& f_{n j l}(r)=\frac{c_{n j l}}{r}\left[(\gamma+\kappa) J_{2 \gamma}(x)-\frac{x}{2} J_{2 \gamma-1}(x)\right], \\
& g_{n j l}(r)=\frac{c_{n j l}}{r} Z \alpha J_{2 \gamma}(x),
\end{aligned}
$$

where

$$
\begin{aligned}
x & =\sqrt{\frac{8 Z r}{a}}, \\
\gamma & =\sqrt{\left(j+\frac{1}{2}\right)^{2}-Z^{2} \alpha^{2}}, \\
\kappa & =(-1)^{j+1 / 2-l}\left(j+\frac{1}{2}\right), \\
c_{n j l} & =\frac{\kappa}{|\kappa|}\left(\frac{1}{Z a \nu^{3}}\right)^{1 / 2},
\end{aligned}
$$

where the J's are Bessel functions, $a$ is the Bohr radius, and $\nu$ is the effective principal quantum number $\left(E_{n l}=-13.6 \mathrm{eV} / \nu^{2}\right)$. To avoid confusion, note that $l$ here is the orbital angular momentum of the electron, rather than the nucleus, as used in Sec. III. Also, the $\kappa$ used here is distinct from the $\kappa$ defined in Eq. (15). Carrying out the integration in Eq. (49), we obtain

$$
\begin{aligned}
T= & \frac{192(-1)^{j_{2}-j_{1}+1} Z^{2}}{a^{4} \nu_{1}^{3 / 2} \nu_{2}^{3 / 2}} \frac{\Gamma\left(-3+\gamma_{1}+\gamma_{2}\right)}{\Gamma\left(4+\gamma_{1}+\gamma_{2}\right) \Gamma\left(4+\gamma_{1}-\gamma_{2}\right) \Gamma\left(4-\gamma_{1}+\gamma_{2}\right)} \\
\times & \left\{\left(3+\gamma_{1}-\gamma_{2}\right)\left(3-\gamma_{1}+\gamma_{2}\right)\left(3+\gamma_{1}+\gamma_{2}\right)\left(2+\gamma_{1}+\gamma_{2}\right)\right. \\
& -5\left(\gamma_{1}+\kappa_{1}\right)\left(3-\gamma_{1}+\gamma_{2}\right)\left(3+\gamma_{1}+\gamma_{2}\right)-5\left(\gamma_{2}+\kappa_{2}\right)\left(3+\gamma_{1}-\gamma_{2}\right)\left(3+\gamma_{1}+\gamma_{2}\right) \\
& \left.+30\left[\left(\gamma_{1}+\kappa_{1}\right)\left(\gamma_{2}+\kappa_{2}\right)+(Z \alpha)^{2}\right]\right\}
\end{aligned}
$$

where $\Gamma$ is the gamma function.

To illustrate the numerical values involved, we will evaluate a value of the matrix element for an electron's interaction with the single particle octupole moment for ${ }^{209} \mathrm{Bi}$. This atom 
has one $6 p_{3 / 2}$ electron above closed subshells $\left(\left(6 p_{1 / 2}\right)^{2}\left(6 s_{1 / 2}\right)^{2} \ldots\right)$. We will consider mixing between $d_{5 / 2}$ and $p_{3 / 2}$ states, each with an angular momentum projection of $3 / 2$. Using table प and Eqs. (48) and (52) gives

$$
\left\langle n_{1} p_{3 / 2}, m=3 / 2\left|U_{\text {oct }}\right| n_{2} d_{5 / 2}, m=3 / 2\right\rangle \approx 1.9 \times 10^{-13}\left(\nu_{1} \nu_{2}\right)^{-3 / 2} \eta \mathrm{cm}^{-1} .
$$

We now compare this with the corresponding matrix element for the interaction with a nuclear magnetic quadrupole moment (MQM), which is another possible source of an atomic EDM. The MQM induced by the T- and P-odd interaction (1) was calculated in Ref. [5], as well as the matrix element of the interaction between an electron and the MQM field. For ${ }^{209} \mathrm{Bi}$ the MQM is $\approx 4.8 \times 10^{-8} \eta e(\mathrm{fm})^{2}$. We get the following estimate:

$$
\left\langle n_{1} p_{3 / 2}, m=3 / 2\left|U_{\text {quad }}\right| n_{2} d_{5 / 2}, m=3 / 2\right\rangle \approx 1.3 \times 10^{-11}\left(\nu_{1} \nu_{2}\right)^{-3 / 2} \eta \mathrm{cm}^{-1} .
$$

By comparing the values of these matrix elements it can be seen that the atomic EDM induced by a nuclear EOM will be less than about $1 \%$ of that induced by a nuclear magnetic quadrupole moment. This same result held for the other atoms and mixing states that we considered. Another mechanism which could produce an atomic EDM is the nuclear Schiff moment, which for heavy atoms (which we are considering) gives a contribution comparable to that of the MQM [5]. Because of this we can conclude that the atomic EDM induced by a single particle nuclear EOM is negligible in comparison with other possible mechanisms. (See the appendix for a discussion of the relative contributions of the octupole, magnetic quadrupole, and Schiff moments.)

Now we will give example values of the matrix elements for those nuclei having a static octupole deformation. As well as possibly having collective EOMs (as shown in Sec. 『V), these nuclei can also have collective MQMs that are an order of magnitude larger than the single particle MQMs discussed above [24]. We will use ${ }^{225} \mathrm{Ac}$ as an example and once again we will consider mixing between $d_{5 / 2}$ and $p_{3 / 2}$ states, each having an angular momentum projection of 3/2. This nucleus has an MQM of $\sim 2 \times 10^{-7} \eta e(\mathrm{fm})^{2}$ [24. We obtain

$$
\left|\left\langle n_{1} d_{5 / 2}, m=3 / 2\left|U_{\text {oct }}\right| n_{2} p_{3 / 2}, m=3 / 2\right\rangle\right| \approx 1.4 \times 10^{-11}\left(\nu_{1} \nu_{2}\right)^{-3 / 2} \eta \mathrm{cm}^{-1}
$$


and

$$
\left|\left\langle n_{1} d_{5 / 2}, m=3 / 2\left|U_{\text {quad }}\right| n_{2} p_{3 / 2}, m=3 / 2\right\rangle\right| \sim 6 \times 10^{-11}\left(\nu_{1} \nu_{2}\right)^{-3 / 2} \eta \mathrm{cm}^{-1} .
$$

These results show that the contribution of the collective nuclear EOM to the atomic EDM is smaller than that of the collective MQM. This also applies to the other isotopes shown in table II, except for ${ }^{229} \mathrm{~Pa}$. $\left({ }^{223} \mathrm{Rn}\right.$ does have a fairly large collective octupole moment, but since it has a closed electron shell the EOM does not contribute to the atomic EDM.) For ${ }^{229} \mathrm{~Pa}$ the contribution of the EOM may be comparable to that of the MQM, but as stated above, it is not certain that this nucleus has a static octupole deformation.

\section{POSSIBLY ENHANCED MAGNETIC QUADRUPOLE MOMENTS IN NUCLEI WITH AN OCTUPOLE DEFORMATION}

Finally, we discuss a mechanism by which single particle magnetic quadrupole moments could be enhanced in even-odd nuclei with an octupole deformation. We will first consider the MQM of such a nucleus in its intrinsic (body-fixed) frame and then transform the MQM into its laboratory frame.

As in Sec. $\amalg$ the wave function of the external nucleon in the intrinsic frame is $\psi_{+K}\left(\mathbf{r}^{\prime}\right)$, defined by Eqs. (32), (33), and (34). The MQM in the intrinsic frame is then

$$
\begin{aligned}
M_{\mathrm{intr}} & =\left\langle\psi_{+K}\left|\hat{M}_{z^{\prime} z^{\prime}}\right| \psi_{+K}\right\rangle \\
& =2 \gamma\left\langle\psi_{1,+K}\left|\hat{M}_{z^{\prime} z^{\prime}}\right| \psi_{2,+K}\right\rangle,
\end{aligned}
$$

where $\hat{M}$ is the operator for the MQM (see Ref. [5]). Note that the MQM is defined for the maximum value of the projection of the angular momentum onto the $z^{\prime}$-axis, so $K=j=I$ (for the ground state of the rotational band $I=j$ ). Using a result from Ref. [5] we have

$$
M_{\mathrm{intr}}=\gamma \frac{2 I-1}{I+1} \frac{e}{m}(\mu-q) \int R_{1} R_{2} r r^{2} d r
$$

where $\mu$ is the magnetic moment of the external nucleon in nuclear magnetons and $q=0(1)$ for a neutron (proton). It should be noted that this (intrinsic frame) single particle MQM 
differs from the spherical nucleus single particle EOMs and MQMs (considered in Sec. III] and Ref. [5], respectively) in that the former is generated due to the interaction $V_{3}$, coming from the nucleus's octupole deformed shape [see Eqs. (33) and (36)], while the latter is due to the interaction $H_{T P}$ (1). The $H_{T P}$ interaction will come into the current situation when we transform into the lab. frame.

Since we are only working out a rough estimate of the MQM we take the integral in the above equation to be

$$
\int R_{1} R_{2} r r^{2} d r \sim \frac{1}{2} r_{0} A^{1 / 3}
$$

where $r_{0}=1.1 \mathrm{fm}$. For both types of nucleons we have $|\mu-q| \approx 1.8$ and so we can write

$$
\left|M_{\text {intr }}\right| \sim 0.2 A^{1 / 3} \frac{2 I-1}{I+1}|\gamma| e(\mathrm{fm})^{2} .
$$

Now we turn to the MQM in the laboratory frame. The wave function of the nucleus in the lab. frame is (as in Sec. IV) $\psi=\psi^{+}+\alpha \psi^{-}$, where $\psi^{ \pm}$is defined by Eqs. (25) and (24) and $\alpha$ is given by Eq. (27) - this is where the interaction $H_{T P}$ comes into the present situation. The MQM in the lab. frame is then

$$
\begin{aligned}
M_{\mathrm{lab}} & =\left\langle\psi\left|\hat{M}_{z z}\right| \psi\right\rangle \\
& =2 \alpha\left\langle\psi^{+}\left|\hat{M}_{z z}\right| \psi^{-}\right\rangle \\
& =\alpha\left(\left\langle I M K\left|\hat{M}_{z z}\right| I M K\right\rangle-\left\langle I M-K\left|\hat{M}_{z z}\right| I M-K\right\rangle\right) \\
& =2 \alpha\left\langle I M K\left|\hat{M}_{z z}\right| I M K\right\rangle .
\end{aligned}
$$

(Once again, the MQM is defined for $M$, the projection of the angular momentum onto the $z$-axis, equal to $I$.) Note that $\left\langle I M-K\left|\hat{M}_{z z}\right| I M-K\right\rangle=-\left\langle I M K\left|\hat{M}_{z z}\right| I M K\right\rangle$, as $\psi_{ \pm K}=\psi_{1, \pm K} \pm \gamma \psi_{2, \pm K}$ and so $\left\langle\psi_{-K}\left|\hat{M}_{z^{\prime} z^{\prime}}\right| \psi_{-K}\right\rangle=-\left\langle\psi_{+K}\left|\hat{M}_{z^{\prime} z^{\prime}}\right| \psi_{+K}\right\rangle$.

Now consider $\left\langle I M K\left|\hat{M}_{z z}\right| I M K\right\rangle$. We have [from Eq. (24)]

$$
\left\langle I M K\left|\hat{M}_{z z}\right| I M K\right\rangle=\frac{2 I+1}{4 \pi} \int D_{M K}^{I *}(\phi, \theta, 0) \psi_{K}^{*}\left(\mathbf{r}^{\prime}\right) \hat{M}_{z z}(\theta, \phi, 0) \psi_{K}\left(\mathbf{r}^{\prime}\right) D_{M K}^{I}(\phi, \theta, 0) d^{3} r^{\prime} d \Omega .
$$


Transforming $\hat{M}_{z z}$ from the lab. frame to the intrinsic $\left(x^{\prime}, y^{\prime}, z^{\prime}\right)$ frame gives $\hat{M}_{z z}(\theta, \phi, 0)=$ $D_{00}^{2 *}(\phi, \theta, 0) \hat{M}_{z^{\prime} z^{\prime}}\left(\theta^{\prime}, \phi^{\prime}, 0\right)$ (see, e.g., [14). Substituting this into Eq. (63) and using Eq. (57) gives (using a formula for the integral of $D$-functions from [14])

$$
\begin{aligned}
\left\langle I M K\left|\hat{M}_{z z}\right| I M K\right\rangle & =\frac{2 I+1}{4 \pi} M_{\text {intr }} \int D_{M K}^{I *}(\phi, \theta, 0) D_{00}^{2 *}(\phi, \theta, 0) D_{M K}^{I}(\phi, \theta, 0) d \Omega \\
& =M_{\mathrm{intr}}\langle I, M ; 2,0 \mid I, M\rangle\langle I, K ; 2,0 \mid I, K\rangle \\
& =M_{\mathrm{intr}}\langle I, I ; 2,0 \mid I, I\rangle^{2} \\
& =\frac{I(2 I-1)}{(I+1)(2 I+3)} M_{\text {intr }} .
\end{aligned}
$$

(Recall that $M=K=I$.) Using Eq. (62) then gives

$$
M_{\mathrm{lab}}=2 \alpha \frac{I(2 I-1)}{(I+1)(2 I+3)} M_{\mathrm{intr}}
$$

and so [using Eq. (61)]

$$
\left|M_{\mathrm{lab}}\right| \sim 0.4 A^{1 / 3}|\alpha||\gamma| \frac{I(2 I-1)^{2}}{(I+1)^{2}(2 I+3)} e(\mathrm{fm})^{2} .
$$

Now we have $|\alpha| \sim 7 \times 10^{-7} \eta$ and $|\gamma| \sim 0.1$ (see Sec. 『V). For $A \approx 225$ we have

$$
\left|M_{\text {lab }}\right| \sim 6 \times 10^{-8} \eta e(\mathrm{fm})^{2} .
$$

This is smaller than the collective MQM due to the spin hedgehog mechanism $-\sim 2 \times$ $10^{-7} \eta e(\mathrm{fm})^{2}$ [24 and so it is not the dominant mechanism. It is, in fact, of the same order of magnitude as the single particle MQM [e.g., for ${ }^{209} \mathrm{Bi}$, approximately $4.8 \times 10^{-8} \eta e(\mathrm{fm})^{2}$ (see Sec. Đ)]. This means that there is no enhancement.

Enhancement was a possibility here due to the relatively large value of $\alpha$ that comes from the smallness of the energy splitting between members of the parity doublet $\left(E_{+}-E_{-}\right)$. However, the inclusion of the factors of $|\gamma| \sim 0.1$ and $I(2 I-1) /[(I+1)(2 I+3)] \sim 0.3$ (this enters on transforming to the lab. frame) ensures that the possible enhancement is not realised.

However, ${ }^{229} \mathrm{~Pa}$ may be an exception to this, as it has a large value of $\alpha$, but it must be remembered that it is not certain that this nucleus has a static octupole deformation (see 
Sec. [V). If is does have such a deformation then its MQM due to the present mechanism would be $\left|M_{\text {lab }}\right| \sim 3 \times 10^{-7} \eta e(\mathrm{fm})^{2}$ [using Eq. (66), the value of $\alpha$ given in Ref. 9], and $|\gamma| \sim 0.1$, which is of the same order of magnitude as the collective MQM due to the spin hedgehog mechanism.

Note that the enhancement of MQMs in deformed nuclei with opposite parity levels close to each other has also been considered in Refs. [四,5].

\section{ACKNOWLEDGMENTS}

One of us (DWM) is grateful to G.F. Gribakin for helpful discussions. This work was supported by the Australian Research Council and by the National Science Foundation through a grant for the Institute for Theoretical Atomic and Molecular Physics at Harvard University and the Smithsonian Astrophysical Observatory.

\section{APPENDIX A: THE RELATIVE CONTRIBUTIONS OF VARIOUS NUCLEAR MOMENTS TO THE ATOMIC ELECTRIC DIPOLE MOMENT}

An atomic electric dipole moment can be generated by T-,P-odd nuclear moments. However, according to the Purcell-Ramsey-Schiff theorem [25,26], a nuclear EDM cannot generate an atomic EDM. This happens because the electrostatic potential of a nucleus is screened by its atomic electrons. Therefore we must look to higher order moments.

The (screened) electrostatic potential of the nucleus can be written as (see, e.g., [5,8,9])

$$
\phi(\mathbf{R})=\int \frac{e \rho(\mathbf{r})}{|\mathbf{R}-\mathbf{r}|} d^{3} r+\frac{1}{Z}(\mathbf{d} \cdot \boldsymbol{\nabla}) \int \frac{\rho(\mathbf{r})}{|\mathbf{R}-\mathbf{r}|} d^{3} r
$$

where $\rho(\mathbf{r})$ is the nuclear charge density $\left(\int \rho(\mathbf{r}) d^{3} r=Z\right), \mathbf{d}=\int e \mathbf{r} \rho(\mathbf{r}) d^{3} r$ is the nuclear EDM, and $\nabla_{i} \equiv \partial_{i} \equiv \frac{\partial}{\partial R_{i}}$. The first non-zero T-,P-odd term in this potential is [5,8,9]

$$
\begin{aligned}
\phi^{(3)} & =-\frac{1}{6} \int e \rho(\mathbf{r}) r_{i} r_{j} r_{k} d^{3} r \partial_{i} \partial_{j} \partial_{k} \frac{1}{R}+\frac{1}{2 Z} \int \rho(\mathbf{r}) r_{i} r_{j} d^{3} r(\mathbf{d} \cdot \nabla) \partial_{i} \partial_{j} \frac{1}{R} \\
& =-\frac{1}{6} \int e \rho(\mathbf{r})\left[r_{i} r_{j} r_{k}-\frac{1}{5} r^{2}\left(r_{i} \delta_{j k}+r_{j} \delta_{i k}+r_{k} \delta_{i j}\right)\right] d^{3} r \partial_{i} \partial_{j} \partial_{k} \frac{1}{R}
\end{aligned}
$$




$$
\begin{aligned}
& -\frac{1}{30} \int e \rho(\mathbf{r}) r^{2}\left(r_{i} \delta_{j k}+r_{j} \delta_{i k}+r_{k} \delta_{i j}\right) d^{3} r \partial_{i} \partial_{j} \partial_{k} \frac{1}{R} \\
& +\frac{1}{2 Z} \int \rho(\mathbf{r})\left(r_{i} r_{j}-\frac{1}{3} r^{2} \delta_{i j}\right) d^{3} r(\mathbf{d} \cdot \boldsymbol{\nabla}) \partial_{i} \partial_{j} \frac{1}{R} \\
& +\frac{1}{6 Z} \int \rho(\mathbf{r}) r^{2} \delta_{i j} d^{3} r(\mathbf{d} \cdot \nabla) \partial_{i} \partial_{j} \frac{1}{R} \\
= & -\frac{1}{6} O_{i j k} \partial_{i} \partial_{j} \partial_{k} \frac{1}{R}+\frac{1}{e} \frac{1}{2 Z} Q_{i j}(\mathbf{d} \cdot \boldsymbol{\nabla}) \partial_{i} \partial_{j} \frac{1}{R} \\
& -\frac{1}{10} \int e \rho(\mathbf{r}) r^{2} r_{i} d^{3} r \partial_{i} \delta_{j k} \partial_{j} \partial_{k} \frac{1}{R}+\frac{1}{6 Z} \int \rho(\mathbf{r}) r^{2} d^{3} r(\mathbf{d} \cdot \boldsymbol{\nabla}) \delta_{i j} \partial_{i} \partial_{j} \frac{1}{R} \\
= & \phi_{\text {octupole }}^{(3)}+\phi_{\text {Schiff }}^{(3)},
\end{aligned}
$$

where

$$
\begin{aligned}
\phi_{\text {octupole }}^{(3)} & =-\frac{1}{6} O_{i j k} \partial_{i} \partial_{j} \partial_{k} \frac{1}{R}+\frac{1}{e} \frac{1}{2 Z} Q_{i j} d_{k} \partial_{i} \partial_{j} \partial_{k} \frac{1}{R} \\
& \approx-\frac{1}{6} O_{i j k} \partial_{i} \partial_{j} \partial_{k} \frac{1}{R} \\
\phi_{\text {Schiff }}^{(3)} & =-\mathbf{S} \cdot \nabla \nabla^{2} \frac{1}{R}=4 \pi \mathbf{S} \cdot \nabla \delta(R)
\end{aligned}
$$

(using $\left.\delta_{j k} \partial_{j} \partial_{k} R^{-1}=\nabla^{2} R^{-1}=-4 \pi \delta(R)\right)$. $Q_{i j}$ is the T,P-even electric quadrupole moment: $Q_{i j}=\int e \rho(\mathbf{r})\left(r_{i} r_{j}-\frac{1}{3} r^{2} \delta_{i j}\right) d^{3} r$. The vector $\mathbf{S}$ is the nuclear Schiff moment and the rank 3 tensor $O_{i j k}$ is the nuclear electric octupole moment. These are given by

$$
\begin{aligned}
O_{i j k} & =\int e \rho(\mathbf{r})\left[r_{i} r_{j} r_{k}-\frac{1}{5} r^{2}\left(r_{i} \delta_{j k}+r_{j} \delta_{i k}+r_{k} \delta_{i j}\right)\right] d^{3} r \\
\mathbf{S} & =\frac{1}{10}\left(\int e \rho(\mathbf{r}) r^{2} \mathbf{r} d^{3} r-\frac{5}{3} \mathbf{d} \frac{1}{Z} \int \rho(\mathbf{r}) r^{2} d^{3} r\right) .
\end{aligned}
$$

The second term in the octupole potential (A3) comes from the screening of the nucleus's Coulomb field. Because only the non-spherically symmetric part of the density will give a non-zero value of $Q_{i j}$, only the external proton contributes to this screening term (in the case of a deformed nucleus all the protons in the external shell contribute). This, together with the presence of the factor $1 / Z$, means that it will be small and so we neglect it. The screening term in the Schiff moment [the second term of Eq. (A6)] is not negligible as the whole density $\rho(\mathbf{r})$ contributes to it and so all of the protons are involved.

$\phi_{\text {Schiff }}^{(3)}$ and $\phi_{\text {Octupole }}^{(3)}$ both appear at the same order in the expansion of Eq. (A1), i.e., both potentials contain third derivatives of $1 / R$ and their corresponding nuclear moments 
are both integrals with integrands containing $r^{3}$ terms. Therefore, we should expect the contributions of the Schiff and octupole moments to the atomic EDM to be roughly the same. However, since the octupole moment is a rank 3 tensor, as opposed to the (vector) Schiff moment, an electron interacting with an octupole moment must have a higher angular momentum (see Sec. $\nabla$ ) than it would need for a Schiff moment. This results in the octupole moment's contribution to the atomic EDM being smaller than the Schiff moment's, as a higher angular momentum wave function penetrates the region close to the nucleus less due to the greater centrifugal barrier. This is confirmed by the calculations in this paper (see Sec. V).

An atomic EDM can also be caused by a nuclear magnetic quadrupole moment (MQM). The vector potential due to such a MQM is 5

$$
A_{i}(\mathbf{R})=-\frac{1}{6} \varepsilon_{i l n} M_{k n} \partial_{l} \partial_{k} \frac{1}{R}
$$

where $M_{k n}$ is the (rank 2) magnetic quadrupole moment tensor:

$$
M_{k n}=-\int\left(r_{k} \varepsilon_{n p q}+r_{n} \varepsilon_{k p q}\right) j_{p} r_{q} d^{3} r
$$

( $j_{p}$ is the electromagnetic current.) Note that this is a lower order term than the Schiff and octupole moments — the potential contains second derivatives of $1 / R$ and the integrand in Eq. (A8) contains $r^{2}$ terms. However, the contribution of the MQM to the atomic EDM will actually be of roughly the same order of magnitude as that of the Schiff and octupole moments. To see this, consider the (nonrelativistic) interaction between the atomic electron's magnetic dipole moment and the magnetic field from the MQM: $\mu_{e} B=\mu_{e}(\boldsymbol{\nabla} \times \mathbf{A})$, where $\mu_{e}$ is the Bohr magneton. Now from Eq. (A7) we have $\boldsymbol{\nabla} \times \mathbf{A} \sim \boldsymbol{\nabla} \times\left(M / R^{3}\right) \sim M / R^{4}$, where $M$ is the magnetic quadrupole moment. From Ref. [5] we have $M \sim \mu_{N} \xi$, where $\mu_{N}$ is the nuclear magneton and $\xi$ is defined by Eq. (3). Therefore we have (in $\hbar=c=1$ units)

$$
\mu_{e} B \sim \frac{\mu_{e} \mu_{N}}{R^{4}} \xi \sim \frac{e}{m_{e}} \frac{e}{m_{p}} \frac{1}{R^{4}} \xi
$$

Now we compare this with the electron's interaction with the octupole: 


$$
e \phi_{\text {octupole }}^{(3)} \sim \frac{e \mathcal{O}}{R^{4}} \sim \frac{e^{2} R_{N}{ }^{2}}{R^{4}} \xi,
$$

using the result for the octupole moment in Eq. (21) $\left(R_{N}=1 \cdot 1 A^{1 / 3} \mathrm{fm}\right.$ is the radius of the nucleus). Therefore, the ratio of the contribution of the octupole moment to the atomic EDM to the contribution of the MQM is $\sim R_{N}^{2} m_{e} m_{p} \sim 10^{-2} A^{2 / 3}$. A more accurate estimate that takes into account the electron angular momenta dependence of the matrix elements gives a ratio that is closer to $10^{-4} A^{2 / 3}$. In Sec. $\nabla$ the ratio was $\sim 0.01$, which is consistent with this result. 


\section{REFERENCES}

[1] J.H. Christenson, J.W. Cronin, V.L. Fitch, and R. Turlay, Phys. Rev. Lett. 13, 138 (1964).

[2] G. Feinberg, Trans. N.Y. Acad. Sci., Ser. II 38, 26 (1977).

[3] P.V. Coveney and P.G.H. Sandars, J. Phys. B 16, 3727 (1983).

[4] W.C. Haxton and E.M. Henley, Phys. Rev. Lett. 51, 1937 (1983).

[5] O.P. Sushkov, V.V. Flambaum, and I.B. Khriplovich, Zh. Éksp. Teor. Fiz. 87, 1521 (1984) [Sov. Phys. JETP 60, 873 (1984)].

[6] V.V. Flambaum, I.B. Khriplovich, and O.P. Sushkov, Nucl. Phys. A 449, 750 (1986).

[7] I.B. Khriplovich, Parity Nonconservation in Atomic Phenomena (Gordon and Breach, Philadelphia, 1991).

[8] N. Auerbach, V.V. Flambaum, and V. Spevak, Phys. Rev. Lett. 76, 4316 (1996).

[9] V. Spevak, N. Auerbach, and V.V. Flambaum, Phys. Rev. C (submitted).

[10] E.M. Henley, Annu. Rev. Nucl. Sci. 19, 367 (1969).

[11] L.D. Landau and E.M. Lifshitz, Quantum Mechanics-Non-relativistic Theory (Pergamon Press, Oxford, 1965).

[12] I. Ahmad and P.A. Butler, Annu. Rev. Nucl. Part. Sci. 43, 71 (1993).

[13] O.P. Sushkov and V.V. Flambaum, Zh. Éksp. Teor. Fiz. 75, 1208 (1978) [Sov. Phys. JETP 48, 608 (1978)].

[14] D.A. Varshalovich, A.N. Moskalev, and V.K. Khersonskii, Quantum Theory of Angular Momentum (World Scientific, Singapore, 1988).

[15] V.B. Berestetskiı̌, E.M. Lifshitz, and L.P. Pitaevskiǐ, Relativistic Quantum Theory (Pergamon Press, Oxford, 1971). 
[16] A. Bohr and B. Mottelson, Nuclear Structure (Benjamin, New York, 1975), Vol. 2.

[17] G.A. Leander and Y.S. Chen, Phys. Rev. C 37, 2744 (1988).

[18] S. Ćwiok and W. Nazarewicz, Nucl. Phys. A 529, 95 (1991).

[19] P.A. Butler and W. Nazarewicz, Rev. Mod. Phys. 68, 349 (1996).

[20] I. Ahmad, J.E. Gindler, R.R. Betts, R.R. Chasman, and A.M. Friedman, Phys. Rev. Lett. 49, 1758 (1982).

[21] V. Grafen, B. Ackermann, H. Baltzer et al., Phys. Rev. C 44, R1728 (1991).

[22] A.I. Levon, J. de Boer, G. Graw et al., Nucl. Phys. A 576, 267 (1994).

[23] J.D. Jackson, Classical Electrodynamics (Wiley, New York, 1975).

[24] V.V. Flambaum, Phys. Lett. B 320, 211 (1994).

[25] E.M. Purcell and N.F. Ramsey, Phys. Rev. 78, 807 (1950).

[26] L.I. Schiff, Phys. Rev. 132, 2194 (1963). 


\section{TABLES}

\begin{tabular}{lcr}
\hline \hline Nucleus & Proton state & $\mathcal{O}_{\text {sing }}\left[\eta e(\mathrm{fm})^{3}\right]$ \\
\hline${ }^{209} \mathrm{Bi}$ & $h_{9 / 2}$ & $1.2 \times 10^{-7}$ \\
${ }^{133} \mathrm{Cs}$ & $g_{7 / 2}$ & $7 \times 10^{-8}$ \\
${ }^{127} \mathrm{I}$ & $d_{5 / 2}$ & $-1.3 \times 10^{-7}$ \\
\hline \hline
\end{tabular}

TABLE I. Approximate values of the unpaired proton octupole moment $\left(\mathcal{O}_{\text {sing }}\right)$ for various nuclei with $I \geq 3 / 2$, in terms of the parameter $\eta$.

\begin{tabular}{lcr}
\hline \hline Nucleus & $I$ & $\left|\mathcal{O}_{\text {coll }}\right|\left[\eta e(\mathrm{fm})^{3}\right]$ \\
\hline${ }^{223} \mathrm{Ra}$ & $\frac{3}{2}$ & $2 \times 10^{-6}$ \\
${ }^{223} \mathrm{Rn}$ & $\frac{7}{2}$ & $7 \times 10^{-5}$ \\
${ }^{225} \mathrm{Ac}$ & $\frac{3}{2}$ & $7 \times 10^{-6}$ \\
${ }^{223} \mathrm{Fr}$ & $\frac{3}{2}$ & $4 \times 10^{-6}$ \\
${ }^{229} \mathrm{~Pa}$ & $\frac{5}{2}$ & $3 \times 10^{-4}$ \\
\hline \hline
\end{tabular}

TABLE II. Approximate values of the collective octupole moment $\left(\mathcal{O}_{\text {coll }}\right)$ for various nuclei that have a static octupole deformation and $I \geq 3 / 2$, in terms of the parameter $\eta$. 